Proof. Apply Theorem J for the case $n=r$. We obtain for $g_{r}$ an expression which differs from the one just written only in the fact that the terms $B_{r, r+1} f_{r+1}+B_{r, r+2} f_{r+2}+\cdots+B_{r, n} f_{n}$ are missing from its numerator. But the coefficients $B_{r, s}=0$ when $r<s$. Hence the two expressions are equal.

REMARK. The generalization of the method to orthonormalization with respect to a general norming or weight function $p$ is obvious. One applies the process described to the functions $(p)^{1 / 2} f_{i}$ and obtains functions $g_{i}$ ( $\equiv$ linear combinations of the $(p)^{1 / 2} f_{i}$ ) which are orthonormal with respect to the weight function unity. Dividing through by the common factor $(p)^{1 / 2}$ one forms functions $g_{i}(p)^{-1 / 2}$ which are orthonormal with respect to $p$.

Carnegie Institute of Technology

\title{
VERTICES OF PLANE CURVES
}

\section{S. B. JACKSON}

1. Introduction. The Four-Vertex Theorem, proved first by Mukhopadhyaya [1], ${ }^{1}$ states that on any oval, not a circle, there are at least four vertices, that is, extrema of the curvature. This result was extended by Fog [2] and Graustein [3] to any simple closed curve with continuous curvature. The discussion by Graustein makes it clear that the Four-Vertex Theorem is valid also for a very large number of non-simple curves. Indeed the class of curves having only two vertices is relatively quite small. The main object of the present paper is to characterize geometrically, as far as possible, the curves with just two vertices. It is thus a proof of the Four-Vertex Theorem by exclusion.

Since a curve with just two vertices consists of two arcs of monotone curvature, a study is made of such arcs ( $\$ 2)$. The most useful fact is that this monotone character of an arc is invariant under direct circular transformations. The property that a point be a vertex of a curve is similarly invariant. This makes it possible to simplify many of the discussions by suitably chosen transformations. Monotone arcs are found to be essentially simple and possess a spiral character.

The existence of vertices on certain types of arcs is established ( $\$ 4)$

Presented to the Society, April 29, 1944; received by the editors February 15, 1944.

${ }^{1}$ Numbers in brackets refer to the Bibliography at the end of the paper. 
and these results applied to give two new proofs of the theorem of Fog and Graustein mentioned above. It is then possible to obtain five results describing the structural character of curves with just two vertices (Theorem 5.1).

It is well known that an oval which meets any circle at most four times has exactly four vertices $[4,5]$. This result is generalized to simple closed curves (Theorem 6.1), and two other results are obtained concerning the relation of such a curve to its extreme circles of curvature (\$6). Another familiar result on ovals, namely that an oval which meets a circle $2 n$ times has at least $2 n$ vertices [6], is also extended to simple closed curves $(\$ 7)$. In this case an additional condition on the cyclic character of the points of intersection is necessary since the curve need not be convex.

At several points in the discussion it is convenient to make use of the following result (Lemma 3.1), which is of some interest in its own right. If a Jordan curve bounding a simply connected region is divided in any manner into three arcs, there exists a circle interior to the region and having points in common with all three arcs.

2. Vertices, and arcs of monotone curvature. By a vertex of a curve of class $C^{\prime \prime}$ is meant a point or a circular arc of the curve for which the curvature is a relative extremum with respect to the neighboring arcs. The term relative extremum is to be understood in the following sense. If $1 / A$ denotes the curvature at the given point (or arc) and $1 / R$ the curvature at an arbitrary point, then in a sufficiently small neighborhood of the point (arc) $1 / R-1 / A \geqq 0$ or $\leqq 0$. The equality sign may be valid at any number of points on the adjacent arcs, but is not identically true for either of them.

An arc on which the curvature is monotone nondecreasing or monotone nonincreasing has no vertices, and conversely, an arc with no vertices has this property. For brevity such arcs will be referred to as monotone arcs. The term arc in this paper is used to denote a map of a line segment which is locally topological but which is not necessarily simple in the large. Thus any segment of a curve will be referred to as an arc of the curve. An arc without double points is a simple arc. In the following discussion all arcs and curves will be understood to be of class $C^{\prime \prime}$ unless the contrary is expressly stated.

Lemma 2.1. Let two curves, $C_{1}$ and $C_{2}$, be tangent in the same direction at a point or coincide along an arc, but not coincide along the neighboring arcs. If $1 / R_{i}$ denotes the curvature of $C_{i}$ and if $1 / R_{1} \geqq 1 / R_{2}$ in a neighborhood of the given point or arc, then in a sufficiently small neighborhood $C_{1}$ lies entirely to the left of $C_{2}$ except for the given point or arc of contact. 
Let $P_{0}$ be the given point or the last point of the given arc of coincidence, and let $s$ be the arc length on $C_{i}$ measured from $P_{0}$. It will be sufficient to prove the lemma for positive values of $s$, for if the directions on the $C_{i}$ are reversed the same proof will then apply to the arcs in that direction. If $P_{0}$ is taken as the origin of coordinates and the common directed tangent as the $x$-axis, the curves $C_{i}$ are given locally by the equations

$$
x_{i}=\int_{0}^{s} \cos \phi_{i} d s, \quad y_{i}=\int_{0}^{s} \sin ^{\prime \prime} \phi_{i} d s,
$$

where $\phi_{i}=\int_{0}^{s} d s / R_{i}$.

Since $1 / R_{1} \geqq 1 / R_{2}$ and the equality sign is not identically true, it follows that for all values of $s$ greater than zero

$$
\phi_{1}(s)=\int_{0}^{s} d s / R_{1}>\int_{0}^{s} d s / R_{2}=\phi_{2}(s) .
$$

By (2.1) this implies at once the following relation:

$$
y_{1}=\int_{0}^{\bullet} \sin \phi_{1} d s>\int_{0}^{s} \sin \phi_{2} d s=y_{2} .
$$

Thus if $P_{1}$ and $P_{2}$ denote the points on $C_{1}$ and $C_{2}$ corresponding to the same arc length $s$, then $P_{1} \neq P_{2}$ for all sufficiently small positive $s$. The slope of the line $P_{1} P_{2}$ may be computed from (2.1) and the limit of this slope as $s$ approaches zero found by l'Hospital's Rule,

$$
\begin{aligned}
\lim _{s \rightarrow 0} m_{P_{1} P_{2}} & =\lim _{s \rightarrow 0} \frac{\int_{0}^{s} \sin \phi_{1} d s-\int_{0}^{s} \sin \phi_{2} d s}{\int_{0}^{s} \cos \phi_{1} d s-\int_{0}^{s} \cos \phi_{2} d s}=\lim _{s \rightarrow 0} \frac{\sin \phi_{1}-\sin \phi_{2}}{\cos \phi_{1}-\cos \phi_{2}} \\
& =\lim _{s \rightarrow 0} \frac{2 \cos 2^{-1}\left(\phi_{1}+\phi_{2}\right) \sin 2^{-1}\left(\phi_{1}-\phi_{2}\right)}{-2 \sin 2^{-1}\left(\phi_{1}+\phi_{2}\right) \sin 2^{-1}\left(\phi_{1}-\phi_{2}\right)}=\infty .
\end{aligned}
$$

By taking $s$ sufficiently small the lines $P_{1} P_{2}$ can be made as nearly parallel to the $y$-axis as we desire. But since $C_{1}$ and $C_{2}$ are both perpendicular to the $y$-axis at $P_{0}$, it is clear that for sufficiently small $s$ the lines $P_{1} P_{2}$ meet each $C_{i}$ only once in this neighborhood. Inasmuch as $P_{1} \neq P_{2}, C_{1}$ and $C_{2}$ cannot meet in this neighborhood. Since by (2.2), $y_{1}>y_{2}, P_{1}$ lies always above, that is, to the left of $C_{2}$, and the lemma is proved.

COROLlary 2.1.1. In a neighborhood of a vertex of a curve, the circle of curvature lies entirely to the left or entirely to the right of the curve according as the curvature at the vertex is a maximum or a minimum. 
This is an immediate consequence of the lemma with the curve and its circle of curvature taken as the curves $C_{i}$.

COROLLARY 2.1.2. At every interior point (or circular arc) of a monotone arc, $\mathcal{A}$, the arc crosses its circle of curvature. The crossing is from right to left or from left to right according as the curvature is monotone nondecreasing or monotone nonincreasing.

This also is a direct consequence of the lemma if the curves $C_{i}$ are taken as follows. Let one of them be the path traced by a moving point which follows the circle of curvature as it approaches the contact point (or arc) and follows $\mathcal{A}$ as it leaves this contact, while the other is the path traced if the point follows $\mathcal{A}$ as it approaches the contact, and the circle as it leaves it.

Lemma 2.2. If, at every interior point (or circular arc), an arc, $\mathcal{A}$, crosses its circle of curvature, then $\mathcal{A}$ is a monotone arc.

For if $\mathcal{A}$ were not monotone it would have vertices, which is impossible by Corollary 2.1.1. Since Lemma 2.2 and Corollary 2.1.2 completely characterize the monotone arcs and since a point not interior to a monotone arc is necessarily either a vertex or a limit point of vertices, the following result is established by exclusion.

LEMma 2.3. If for a point (or circular arc) interior to an arc, $\mathcal{A}$, the circle of curvature lies locally entirely to one side of $\mathcal{A}$ then this point (or arc) is either a vertex or a limit point of vertices.

LEMMA 2.4. A monotone arc of nondecreasing (nonincreasing) curvature is carried into a monotone arc of nondecreasing (nonincreasing) curvature by any direct circular transformation. $A$ vertex of an arc is carried by a direct circular transformation either into a vertex of the same kind on the transformed arc or into a limit point of such vertices.

This result follows immediately from the fact that Corollary 2.1.1, Corollary 2.1.2, Lemma 2.2, and Lemma 2.3 characterize vertices and monotone arcs in terms that are invariant under direct circular transformations. This fact affords a simple proof of the following well known result.

Lemma 2.5. If $P_{0}$ is any point of a monotone arc $\mathcal{A}$ and $P$ is any point further along $\mathcal{A}$ in the positive direction, the circle of curvature at $P$ lies to the left or to the right of the circle of curvature at $P_{0}$ according as the curvature on $A$ is nondecreasing or nonincreasing. The circles have no point in common unless they are identical and $\mathcal{A}$ contains the circular arc from $P_{0}$ to $P$. 
It is clearly sufficient to prove the lemma for points $P$ in an arbitrarily small neighborhood of $P_{0}$. Suppose the circles of curvature at $P$ and $P_{0}$ have a point $Q$ in common. By a direct circular transformation let $Q$ be carried into the point at infinity. The transformed arc, $A^{\prime}$, then has zero curvature at the transforms of $P_{0}$ and $P$, which, by the monotone character of $\mathcal{A}^{\prime}$, can occur only if the curvature is zero on the entire arc between the two points. The lemma follows by transforming back to $A$. This argument is valid even if $Q$ coincides with $P_{0}$. The fact that the circle of curvature at $P$ lies to the left or right of that at $P_{0}$ according as the curvature is nondecreasing or nonincreasing is a consequence of Corollary 2.1.2.

Corollary 2.5.1. A monotone arc $\mathcal{A}$ is simple unless it contains a complete circle traced one or more times. In this latter case it is tangent to itself without crossing at a single point or along a single arc of this circle.

CoRollary 2.5.2. If a monotone arc, $\mathcal{A}$, has positive curvature, it is an inwinding or an outwinding spiral according as the curvature is nondecreasing or nonincreasing. It is simple except for any complete circles it may contain.

Both corollaries are immediate consequences of the lemma. For Corollary 2.5.2 the spiral character follows from the fact that for a point of positive curvature the inside of the circle of curvature lies to the left of the arc.

3. A lemma on circles. At several points in the remainder of the paper it will be convenient to make use of the following lemma.

LEMMA 3.1. Let $R$ be a closed simply connected region of the plane bounded by a Jordan curve, and let the Jordan curve be divided into three arcs, $\mathcal{A}_{1}, \mathcal{A}_{2}, \mathcal{A}_{3}$. Then there exists a circle contained in $\mathrm{R}$ and having points in common with all three arcs.

The following simple proof is due to Paul Erdös and is given in preference to the more complicated proof originally given by the author.

Let $A_{i}$ be the set of points of $R$ whose distances from arc $A_{i}$ do not exceed their distances from the other $\mathcal{A}$ 's. The sets $A_{i}$ are clearly closed. Moreover they are connected, for if $X, Y \in A_{i}$ and if $P, Q$ are the corresponding nearest points of $\mathcal{A}_{i}$, then the line segments $X P$ and $Y Q$ and the arc $P Q$ of $\mathcal{A}_{i}$ all belong to $A_{i}$. Now $A_{1} \cup A_{2} \cup A_{3}=R$ and since each of the arcs has an end point in common with the other two, $A_{i} \cap A_{j} \neq 0$. Thus $A_{1} \cup A_{2}$ is a closed connected set. 
Suppose $A_{1} \cap A_{2} \cap A_{3}=0$. Then the intersection of $A_{3}$ with $A_{1} \cup A_{2}$ consists of the two disjoint nonvacuous closed sets $A_{1} \cap A_{3}$ and $A_{2} \cap A_{3}$, and is therefore not connected. This is impossible since it contradicts the unicoherence of R. ${ }^{2}$ Thus there is some point $P_{0}$ in $A_{1} \cap A_{2} \cap A_{3}$. By definition, the distances from $P_{0}$ to the three $\mathcal{A}_{i}$ have the same value, say $r$. The circle with center at $P_{0}$ and radius $r$ is the required circle.

COROLLARY 3.1.1. If a simply connected region is bounded by $n$ differentiable arcs $(n \geqq 3)$, and if the angles formed by the arcs interior to the region are all less than or equal to $\pi$, then there exists a circle contained in the region and tangent to at least three of the arcs.

If two consecutive arcs are taken as the $\operatorname{arcs} \mathcal{A}_{1}$ and $\mathcal{A}_{2}$ of Lemma 3.1 and all the other arcs together taken as $\mathcal{A}_{3}$, then by the lemma there is a circle interior to the region and having points in common with at least three of the arcs. If, at their common point, two consecutive arcs make an angle less than $\pi$ interior to the region, there is no circle through this point which lies interior to the region. Such a point thus cannot be on the given circle. But if a circle meets a differentiable arc at an interior point without crossing it, they are tangent, whence all the points common to the circle and the arcs are tangencies. Two of the contact points may coincide, for if two consecutive arcs make an angle of $\pi$ at their common point, the circle may be tangent to them both at this point.

4. Location of vertices on a curve. The following lemma establishes the existence of vertices on certain types of arcs, and is of fundamental importance in the succeeding work.

LEMma 4.1. If a simple, noncircular arc, $A B$, is tangent to a circle (or line) in the same direction at $A$ and $B$, and never crosses this circle (line), there is a maximum or a minimum of curvature interior to $A B$ according as $A B$ lies to the right or left of the given directed circle (line).

It is sufficient to prove the lemma for the case when $A B$ meets the circle only at $A$ and $B$, since otherwise $A B$ may be replaced by a subarc which does have this property. Suppose $A B$ lies to the left of the directed circle. Let a point $P_{0}$ of the directed circular arc $B A$ be carried into the point at infinity by a direct circular transformation. The circle goes into a straight line and the $\operatorname{arc} A B$ into an $\operatorname{arc} A^{\prime} B^{\prime}$ tan-

${ }^{2}$ A continuum, that is, a closed connected set, is said to be unicoherent if, when it is written in any manner as the sum of two continua, $C_{1}$ and $C_{2}$, the set $C_{1} \cap C_{2}$ is also a continuum. This is a well known property of closed simply connected regions of the plane. 
gent to this directed line at $A^{\prime}$ and $B^{\prime}$ and lying to its left. The points $A^{\prime}, B^{\prime}$ appear in that order on the directed line. It is clear, from the Gauss-Bonnet formula, that the angular measure of the $\operatorname{arc} A^{\prime} B^{\prime}$ is zero. Since at $A^{\prime}$ and $B^{\prime}$ the arc lies to the left of the line there are points of positive curvature arbitrarily near $A^{\prime}$ and $B^{\prime}$, but since the angular measure is zero there must be points of negative curvature on $A^{\prime} B^{\prime}$. Thus there is at least one minimum of curvature interior to $A^{\prime} B^{\prime}$. By Lemma 2.4 this implies that there is a minimum interior to $A B$. This completes the proof of the lemma when $A B$ lies to the left of the circle. The other case reduces to this by reversing the direction on the arc.

COROLLARY 4.1.1. If a simple, noncircular arc, $A B$, is tangent to a circle (or line) in the same direction at $A$ and $B$ and lies to the left of the circle (line) near $A$ and $B$ there is at least one minimum of curvature interior to $A B$. If $A B$ lies to the right of the circle near $A$ and $B$ there is at least one maximum of curvature interior to $A B$.

Let $A B$ lie locally to the left of the given circle at $A$ and $B$. By adjoining the directed circle arc, $B A$, to $A B$ a closed curve $\bar{C}$ of class $C^{\prime}$ is obtained. Since, by a direct circular transformation, this can be transformed into a curve where $B A$ is a line segment, the proof will be confined to this case.

If there are points of negative curvature the result is trivial, since there are points of positive curvature arbitrarily close to $A$ and $B$. Suppose then that the curvature is positive and consider the smallest circle containing $\bar{C}$. There is a smallest such circle since $\bar{C}$ is a closed set, and there must be at least two distinct contact points of $\bar{C}$ with this circle, $P$ and $Q$. Neither of the contact points can be on the line segment $B A$ for there would then be points of $\bar{C}$ outside the circle. Arc $A B$ is simple so the tangencies are in the same direction at $P$ and $Q$. Since $A B$ has positive curvature and is interior to the circle, it lies to the left of the directed circle. The arc $P Q$ therefore has a minimum of curvature by Lemma 4.1, or if $P Q$ is a circular arc it is a minimum of curvature itself. This proves the first part of the corollary. The second part may be reduced to this by reversing the direction on $A B$.

An $\operatorname{arc} A B$ for which $A=B$ will be called a closed arc. If it contains no other double points it is a simple closed arc.

Lemma 4.2. A closed arc, not a circle, contains at least one vertex interior to the arc.

For if an arc has no vertices it is monotone, and by Lemma 2.5 the 
first and last points can coincide only if the entire arc is a circle traced one or more times.

Lemma 4.3. Let $A B$ be a simple closed arc, not a circle, bounding a region $R$, and let $\theta$ be the angle interior to $R$ between the two tangents at the double point. If $\theta \leqq \pi$, there is a maximum or a minimum of curvature interior to $A B$ according as $R$ lies to the left or the right of the arc. If $\theta \geqq \pi$, there is a maximum or a minimum of the curvature interior to $A B$ according as $R$ lies to the right or left of the arc.

First let $\theta$ be less than or equal to $\pi$. Let $A B$ be divided into three arcs, $A P, P Q, Q B$, so that $P Q$ is not an arc of a circle. This can be done since $A B$ is not a circle. By Corollary 3.1.1 there is a circle entirely in $R$ and tangent to all three of these arcs. The subarc of $A B$ from the contact point on $A P$ to the contact point on $Q B$ satisfies the conditions of Lemma 4.1, whence the lemma follows at once since the arc is to the right or left of the circle according as $R$ is to the left or right of the arc. The case when $\theta \geqq \pi$ may be reduced at once to the above by a direct circular transformation taking a point of $R$ into the point at infinity. It should be noted that under this transformation $R$ corresponds to the exterior, not the interior of the transformed arc.

When $\theta=\pi$, that is, when the directed tangents at $A$ and $B$ coincide, both parts of Lemma 4.3 apply, and we obtain the following special case.

Corollary 4.3.1. If $A B$ is a simple closed arc, not a circle, and the directed tangents at $A$ and $B$ coincide, there is both a maximum and $a$ minimum of curvature interior to the arc.

Lemma 4.1 is a direct generalization of the results obtained by Graustein [3] on arcs of type $\Omega$. The material of this section also affords an easy proof of the following result of Graustein [3] and Fog [2].

THEOREM 4.1. Every simple closed curve, $C$, not a circle, has at least four vertices.

Let $C$ be directed so that its interior is to the left of the curve, and let $P_{0}$ be the point of absolute minimum curvature. Then $C$, considered as a simple closed arc from $P_{0}$ to $P_{0}$, satisfies the conditions of Lemma 4.3, and so has a minimum interior to the arc. Thus there are two minima of the curvature on $C$, whence there are two maxima and so four vertices.

An alternative proof can be given as follows. Let $C$ be divided into three arcs, none of them merely circular arcs. By Corollary 3.1.1 there 
is a circle entirely inside $C$ and tangent to each of the three arcs. At least two of the contact points are distinct and all tangencies are in the same direction. The arcs into which these contact points divide $C$ both satisfy Lemma 4.1 , whence $C$ has two minima (or two maxima) and thus four vertices.

5. Curves with two vertices. If a curve, $C$, is to have exactly two vertices it must consist of two monotone arcs, whose characteristics were discussed in \$2. Corollary 2.5.1 leads at once to the following result.

Lemma 5.1. A curve, $C$, with exactly two vertices may be divided into two arcs, each of which is simple unless it contains a full circle. In this latter case the arc is tangent to itself, without crossing, either at a single point or along $a$ single arc of the circle.

If, from a curve $C$ with just two vertices, all arcs which are complete circles are deleted, the resulting curve, $\bar{C}$, will still be a closed curve of class $C^{\prime \prime}$. Moreover the operation neither adds nor takes away vertices, whence $\bar{C}$ consists of two simple monotone arcs. The curve $\bar{C}$ is called a normalized curve.

A point where a curve meets itself is called a double point. A double point is called simple if the curve passes through it exactly twice.

Lemma 5.2. A normalized curve, $\bar{C}$, having exactly two vertices has double points, and all the double points are simple.

If there were no double points $\bar{C}$ would be a simple closed curve, which is impossible by Theorem 4.1. If any double point were not simple it would divide $\bar{C}$ into at least three arcs, none of which are circles since $\bar{C}$ is normalized. But by Lemma 4.2 each arc would then contain a vertex, which is impossible.

Let us consider the circle of curvature at the point of minimum curvature on any curve $C$ with two vertices. Since the curve consists of two monotone arcs, it follows from Lemma 2.5 that the entire curve $C$ lies on or to the left of this circle. By a direct circular transformation, a point to the right of this circle may be taken into the point at infinity. $C$ is then carried into a curve $C^{\prime}$ whose curvature is always positive and which is inside the transformed circle. If $M$ and $m$ are the points of maximum and minimum curvature on $C^{\prime}$, all the circles of curvature lie inside that at $m$ and outside that at $M$, by Lemma 2.5. The center of curvature, $O$, at the point $M$ is interior to every circle of curvature. If $P$ is a point moving in the positive direction on $C^{\prime}$ the radius vector, $O P$, always turns in the same direc- 
tion, namely counterclockwise. Thus if $C^{\prime}$ is tangent to itself at any point the directed tangents at this point must coincide.

Lemma 5.3. If a curve, $C$, having exactly two vertices is tangent to itself at any point, the directed tangents coincide.

For otherwise $C^{\prime}$ would also have oppositely directed tangents at a point, and this has just been shown impossible.

A simple closed arc of a curve is called a loop. If the remainder of the curve never crosses this arc the loop is called a simple loop.

Lemma 5.4. A curve, $C$, having exactly two vertices, has exactly two simple loops, one containing each vertex.

Let $C$ be transformed into curve $C^{\prime}$ as in the last proof. It is sufficient to prove the lemma for $C^{\prime}$. We shall proceed to establish the existence of a simple loop containing the maximum of curvature. If the maximum of curvature occurs on a circular arc which contains a full circle, this arc coincides with the circle of maximum curvature. In this case the circle itself constitutes the desired simple loop since it is completely within all other circles of curvature.

Suppose then that the maximum curvature occurs at a point or on an arc less than a full circle. As before let $M$ and $m$ be points of maximum and minimum curvature respectively. By Corollary 2.5.2 the arcs $m M$ and $M m$ are inwinding and outwinding spirals respectively, each arc being simple except for tangencies without crossing which occur whenever a complete circle is contained in the arc. Since by Lemma 5.2 even the normalized curve $\bar{C}$. is not simple it follows that arcs $m M$ and $M m$ intersect. Let $A$ be the first point where $M m$, as it is traced from $M$, meets $m M$. We shall show that $\operatorname{arc} A M A$ is the required simple loop. If $A$ is a double point of $m M, A M$ will mean the arc from $A$ to $M$ which does not pass again through $A$.

If $A M$ were not simple it would contain a full circle which would have $M$ in its interior and $A$ on its exterior, whence $A$ could not be the first point where $M m$ meets $m M$. Similarly $M A$ is simple. Thus $A M A$ is a loop, not a circle, containing the maximum of curvature. Since the entire curve $C^{\prime}$ spirals in the counterclockwise direction about the circle of curvature at $M$, this circle lies interior to and to the left of $A M A$. The angle formed at $A$ interior to the loop is less than $\pi$, for otherwise by Lemma 4.3 the arc contains a minimum of curvature, which is false.

No points of $m M$ lie interior to $A M A$ since by its spiral character it never crosses itself and by construction it never crosses $M A$. Let a moving point $P$ trace $M m$. At $A$ it definitely passes to the exterior of 
the loop. Let $B$ be the first point where $P$ meets the loop again. If $B$ does not exist the loop is simple. If $B$ lies on $M A$ the arc from $B$ to $B$ is a full circle containing the loop in its interior. The outwinding spiral character of $M m$ assures that $P$ can never get to the interior of the loop, so again it is simple. Suppose finally $B$ is on $A M$. The arc $B M A B$ is then a simple closed arc bounding a region to its left. Moreover the angle at $B$ interior to this region is clearly greater than or equal to $\pi$. By Lemma 4.3 this arc would then contain a minimum, which is impossible. Thus the loop $A M A$ is simple. This establishes the existence of a simple loop containing the vertex of maximum curvature. But since a mere reversal of direction on a curve interchanges maximum and minimum curvatures, the same proof applies to the other case.

By Lemma 4.2 every loop, not a circle, contains at least one vertex, whence there can be no other loops on the curve which are not circles. Moreover since every circle in the curve distinct from the above loops is a circle of curvature on a monotone arc, it is crossed by the arc and is not a simple loop. This completes the proof of the lemma.

A closed curve in the plane divides the plane into a certain number of regions bounded by arcs of the curve. The definition of a simple loop implies that the loop constitutes the entire boundary of one of these regions. For the loop containing the maximum point this region was shown above to lie to the left of the loop, while for the loop containing the minimum point the region bounded is to the right. It should be noted that one of the regions bounded by the curve is always an infinite one.

LEMMA 5.5. The region bounded by a simple loop of a curve $C$ with exactly two vertices lies to the right or left of the loop according as this loop contains the minimum or the maximum of curvature.

LEMMA 5.6. If $C$ is a curve with exactly two vertices, the only regions determined by $C$ which are bounded in the same sense by all their bounding arcs are those bounded by the two simple loops.

To prove this, let $C$ be transformed into a curve $C^{\prime}$ of positive curvature as before. The regions bounded by the two loops in this case are the infinite region and the region containing the center of curvature, $O$, at $M$. Let $P$ be a point of any other region and consider the ray $O P$. By the spiral character of $C^{\prime}$, ray $O P$ always crosses it in the same direction, namely from left to right. Point $P$ is thus to the right of one of the arcs bounding its region and to the left of another since there is at least one crossing between $O$ and $P$ and another between $P$ 
and the point at infinity. This establishes the lemma for $C^{\prime}$ and thus for $C$.

The results of this section may be summarized in the following theorem.

Theorem 5.1. A curve, $C$, with exactly two vertices, has the following properties:

(a) The corresponding normalized curve, $\bar{C}$, may be divided into two simple arcs;

(b) The corresponding normalized curve, $\bar{C}$, must contain double points, and all the double points are simple;

(c) The curve, $C$, has exactly two simple loops, one containing the maximum of curvature and bounding a region to its left, the other containing the minimum of curvature and bounding a region to its right;

(d) No region determined by $C$, other than those mentioned in (c), is bounded in the same sense by all its bounding arcs;

(e) At any point where $C$ is tangent to itself the directed tangents coincide.

6. Curves with four vertices. It is a well known theorem that an oval which has at most four intersections with any circle has exactly four vertices $[4,5]$. This result may be generalized as follows.

Theorem 6.1. A simple closed curve, $C$, which meets any circle or straight line at most four times has exactly four vertices.

Let the curve be directed so the interior lies to its left. First let us show that the circle of curvature at any point (or arc) of maximum curvature on $C$ can have no further points in common with the curve. In a neighborhood of the given vertex the circle is interior to $C$ by Corollary 2.1.1. In this neighborhood let points $P$ and $Q$ of the circle be chosen on opposite sides of the vertex. Suppose the circle contains a point $R$ outside $C$. Now let the curvature of the circle decrease, keeping it tangent to $C$ at the maximum point, $M$, so that the new circle lies to the right of the original one. This deformation may be taken so small that the points $P, Q, R$ do not cross $C$. But by Lemma 2.1 the new circle lies to the right of $C$ in a neighborhood of $M$. Thus, in addition to $M$ itself, there are crossings on $M P, P R, R Q$, and $Q M$. This contradicts the assumption of at most four intersections. If the circle of curvature at $M$ contains no exterior points but does contain a point of tangency with $C$, the curve lies to the right of the directed circle at this point, $R$. The deformation above then takes $R$ to a point outside $C$ and the contradiction is obtained as before.

Suppose Theorem 6.1 is false, that is, suppose $C$ has at least six 
vertices. If $M_{1}, M_{2}, M_{3}$ are three maximum points, let $C$ be divided into arcs $M_{1} M_{2}, M_{2} M_{3}, M_{3} M_{1}$. By Corollary 3.1.1 there is a circle interior to $C$ and tangent to all three arcs. None of the points $M_{i}$ can be points of contact since we have shown that the largest circles at the $M_{i}$ which are locally interior to $C$ can never meet $C$ again. Thus there are three distinct points of contact, $C_{1}, C_{2}, C_{3}$. A slightly larger concentric circle will then have the $M_{i}$ outside and the $C_{i}$ inside, contradicting the hypothesis of only four intersections. Thus the assumption is false and the theorem is proved.

THEOREM 6.2. If a simple closed curve, $C$, has exactly four vertices, the circles of curvature at the vertices have no further points in common with $C$.

Let $M$ be a point of maximum curvature and suppose the circle of curvature at $M$ meets $C$ at a point other than this vertex. Consider the family of circles tangent to $C$ at $M$ and lying on or to the left of the circle of curvature at $M$. Since the circles of this family which have sufficiently small radius meet $C$ only at $M$, while the circle of curvature does meet $C$ at some other point, there is a circle of the family lying entirely inside $C$ but tangent to it at some point $P$ distinct from $M$. Since $C$ is simple, the circle is tangent to $C$ in the same direction at $P$ and $M$, lying to the left of $C$ at both points. The arcs $M P$ and $P M$ then each contain a maximum of curvature by Lemma 4.1. This gives three maximum points and thus at least six vertices. This contradicts the hypothesis of only four vertices so the circle of curvature cannot meet $C$ again. Since a reversal of direction on $C$ interchanges maximum and minimum points, the same proof shows that the circles of curvature at the minimum points also do not meet the curve again.

TheOREM 6.3. If a simple closed curve, $C$, has exactly four vertices, a necessary and sufficient condition that it can be carried into an oval by a direct circular transformation is that either the two maximum or the two minimum circles of curvature intersect.

The term oval here means a simple closed curve with nonvanishing curvature. If an oval with exactly four vertices is directed so the curvature is positive, the entire curve lies inside the circles of curvature at the points of minimum curvature by Theorem 6.2. Thus each such circle contains an arc of the other near the opposite minimum point, and they must intersect. The condition of the theorem is thus necessary, since the properties in question are preserved by direct circular transformations. 
Consider now a simple closed curve, $C$, with just four vertices, having two of its extreme circles of curvature intersecting. The curve may be so directed that these are the circles of curvature at the minimum points. The entire curve then lies to the left of both circles. Since the circles intersect, there is a point to the right of both circles. Let this point be taken into the point at infinity by a direct circular transformation. The two circles of minimum curvature for the new curve now have positive curvature since their interiors lie to their left. But since there are only four vertices, and monotone arcs go into monotone arcs, the curvature is positive at all points. The transformed curve is therefore an oval, and the proof is complete.

7. Curves with $2 n$ vertices. It is a well known fact that an oval which crosses a circle $2 n$ times has $2 n$ vertices [6]. This fact may be generalized to simple closed curves as follows.

Theorem 7.1. Let a simple closed curve, $C$, be met by a circle $\bar{C}$. If, among the arcs into which $\bar{C}$ divides $C$, there are $2 n-1$ arcs $P_{2 i-1} P_{2 i}$ $(i=1,2, \cdots, 2 n-1)$ such that the points $P_{k}$ are in the same cyclic order on $C$ and $\bar{C}$, then $C$ has at least $2 n$ vertices.

The point $P_{2 i}$ may coincide with $P_{2 i+1}$. If $C$ is an oval the condition on the cyclic order of the points is automatically fulfilled. It should be noted that the theorem does not require that the chosen arcs be all the arcs of $C$.

Any one of the chosen arcs lies either wholly inside or wholly outside of $\bar{C}$. There are therefore at least $n$ of these arcs in one place or the other. We may suppose the $n$ arcs interior to $\bar{C}$ since the other case can be reduced to this by a direct circular transformation. Let these $n$ arcs, in order, be denoted by $A_{i}$, their end points by $Q_{i}, R_{i}$, and the arc of $\bar{C}$ from $R_{i}$ to $Q_{i+1}$ by $\bar{C}_{i}$. It may be assumed without loss of generality that none of the points $Q_{i}, R_{i}$, are points of tangency of $C$ and $\bar{C}$, for if they are $\bar{C}$ may be replaced by a slightly smaller concentric circle for which this is not true. This implies that no $\bar{C}_{i}$ reduces to a point. By hypothesis the region interior to $\bar{C}$ bounded by the $\mathcal{A}_{i}$ and the $\bar{C}_{i}$ is bounded in the same sense by all these arcs. By suitably directing $C$ the region may be made to lie to the left of these arcs.

Let $A_{j}$ and $A_{j+1}$ be any two consecutive $\mathcal{A}_{i}$, and consider the region bounded by $A_{j}, A_{j+1}, \bar{C}_{j}$, and the directed arc of $\bar{C}$ from $R_{j+1}$ to $Q_{j}$. This is a simply connected region bounded by differentiable arcs. Let the boundary be divided into three arcs as follows: $\mathcal{A}_{j}, \bar{C}_{j}$, and the remainder of the boundary. By Lemma 3.1 there is a circle in the region having points in common with all three arcs, and these 
are all points of tangency as in Corollary 3.1.1. Since there is a contact point on $\bar{C}_{j}$, there cannot be one on the rest of $\bar{C}$, whence there are contact points on $\mathcal{A}_{j}$ and $\mathcal{A}_{j+1}$ which we shall call $T_{j}$ and $S_{j+1}$ respectively. These points are distinct since $\mathcal{A}_{j}$ and $\mathcal{A}_{j+1}$ have no point in common and are distinct from $R_{j}$ and $Q_{j+1}$ since $C$ is not tangent to $\bar{C}$ at these latter points. The tangencies at $T_{j}$ and $S_{j+1}$ are clearly in the same direction on the circle.

If the same operation is carried out for $\operatorname{arcs} \mathcal{A}_{j-1}$ and $\mathcal{A}_{j}$ we obtain points $T_{j-1}$ and $S_{j}$ on $\mathcal{A}_{j-1}$ and $\mathcal{A}_{j}$ respectively. We shall show that the points $S_{j}, T_{j}$ occur in that order as $A_{j}$ is traced from $Q_{j}$ to $R_{j}$. Let the circles constructed with contact points on $\bar{C}_{j-1}$ and $\bar{C}_{j}$ be denoted by $O_{j-1}$ and $O_{j}$ respectively. Suppose that $\operatorname{arc} Q_{j} T_{j}$ does not contain $S_{j}$, and consider the region bounded by $Q_{j} T_{j}$, the arc of $O_{j}$ from $T_{j}$ to its contact point on $\bar{C}_{j}$, and the arc of $\bar{C}$ from $Q_{j}$ to this same contact point. This region is exterior to $O_{j}$ and contains the $\operatorname{arc} T_{j} S_{j} R_{j}$ by hypothesis. The point of contact of $O_{j-1}$ and $\bar{C}_{j-1}$ is exterior to this region and also exterior to $O_{j}$. Thus each of the arcs of $O_{j-1}$ from its contact point with $\bar{C}_{j-1}$ to $S_{j}$ must cross $O_{j}$ twice. This is impossible, since two distinct circles can meet in only two points. Thus $S_{j}$ and $T_{j}$ occur in that order on $\mathcal{A}_{j}$. The various arcs $T_{j-1} S_{j}$ of the given curve $C$ are therefore distinct arcs with no points in common. But each of these $n$ arcs of $C$ satisfies the conditions of Corollary 4.1.1, and thus contains a maximum point since it lies locally to the right of $O_{j-1}$ at $T_{j-1}$ and $S_{j}$. This proves the existence of $n$ maximum points and thus $2 n$ vertices.

It should be noted that if a simple closed curve intersects a circle an infinite number of times any number of arcs may be found satisfying the conditions of the theorem. The following result then follows at once.

COROLLARY 7.1.1. If a simple closed curve intersects a circle infinitely often, it has an infinite number of vertices.

\section{BIBLIOGRAPHY}

1. S. Mukhopadhyaya, New methods in the geometry of a plane arc, Bull. Calcutta Math. Soc. vol. 1 (1909) pp. 31-37.

2. D. Fog, Über den Vierscheitelsatz und seine Verallgemeinerungen, Sitsungsberichte der Berlin Akademie der Wissenschaft, 1933, pp. 251-254.

3. W. C. Graustein, Extensions of the four-vertex theorem. Trans. Amer. Math. Soc. vol. 41 (1937) pp. 9-23.

4. G. Bol, Ein Satz über Eilinien, Abh. Math. Sem. Hansischen Univ. vol. 13 (1940) pp. 319-320.

5. W. Blaschke, Vorlesungen ïber Differentialgeometrie I, Berlin, 1930, p. 49.

6. - Kreis und Kugel, Leipzig, 1916, p. 161.

UNIVERSITY OF MARYLAND 